In: Advances in Gabor Analysis,

H. G. Feichtinger and T. Strohmer, eds.,

Birkhäuser, Boston, 2003, pp. 153--169.

\title{
Integral Operators, Pseudodifferential Operators, and Gabor Frames
}

\section{Christopher Heil}

\begin{abstract}
This chapter illustrates the use of Gabor frame analysis to derive results on the spectral properties of integral and pseudodifferential operators. In particular, we obtain a sufficient condition on the kernel of an integral operator or the symbol of a pseudodifferential operator which implies that the operator is trace-class. This result significantly improves a sufficient condition due to Daubechies and Hörmander.
\end{abstract}

\subsection{Introduction}

Pseudodifferential operators arise naturally in a variety of areas in mathematics, science, and engineering, including partial differential equations, quantum mechanics, and signal processing. An elegant sketch of how and why pseudodifferential operators occur in these contexts can be found in the recent book of Gröchenig [13, Ch. 14].

The Weyl and Kohn-Nirenberg correspondences are perhaps the two most important methods of constructing pseudodifferential operators. Each correspondence is a linear mapping which associates to a given "symbol function" $\sigma(x, \xi)$ a corresponding operator, often denoted $\sigma(X, D)$, which is obtained from the symbol $\sigma(x, \xi)$ by in some sense formally substituting the position operator $X f(x)=x f(x)$ and the differentiation operator $D f(x)=\frac{1}{2 \pi i} f^{\prime}(x)$ for the variables $x, \xi$ in the symbol. In particular, if $\sigma(x, \xi)=x$ then (using either correspondence) $\sigma(X, D)=X$, and if

$\sigma(x, \xi)=\xi$ then $\sigma(X, D)=D$. In the language of quantum mechanics, the Weyl and Kohn-Nirenberg correspondences are therefore examples of "quantization rules." For extensive and detailed discussions of pseudodifferential operators, we refer to the books of Hörmander [18], Stein [27], Taylor [30], Folland [11], and Wong [32].

We will concern ourselves in this chapter only with the case of squareintegrable symbol functions $\sigma(x, \xi)$. With this restriction, the operator $\sigma(X, D)$ defined by either correspondence is a compact operator mapping $L^{2}(\mathbb{R})$ into itself. We also consider integral operators defined by square- 
integrable kernels $k(x, y)$. To distinguish between these various cases, we introduce the following notation.

Definition 7.1.1. Given a kernel function $k \in L^{2}\left(\mathbb{R}^{2}\right)$, the integral operator determined by $k$ is the operator $A_{k}: L^{2}(\mathbb{R}) \rightarrow L^{2}(\mathbb{R})$ defined by

$$
A_{k} f(x)=\int k(x, y) f(y) d y \text {. }
$$

Given a symbol function $\sigma \in L^{2}\left(\mathbb{R}^{2}\right)$, the Weyl transform of $\sigma$ is the operator $L_{\sigma}: L^{2}(\mathbb{R}) \rightarrow L^{2}(\mathbb{R})$ defined by

$$
L_{\sigma} f(x)=\iint \sigma\left(\frac{x+y}{2}, \xi\right) e^{2 \pi i(x-y) \xi} f(y) d y d \xi .
$$

Given a symbol function $\tau \in L^{2}\left(\mathbb{R}^{2}\right)$, the Kohn-Nirenberg transform of $\tau$ is the operator $K_{\tau}: L^{2}(\mathbb{R}) \rightarrow L^{2}(\mathbb{R})$ defined by

$$
\begin{aligned}
K_{\tau} f(x) & =\int \tau(x, \xi) \hat{f}(\xi) e^{2 \pi i x \xi} d \xi \\
& =\iint \tau(x, \xi) e^{2 \pi i(x-y) \xi} f(y) d y d \xi
\end{aligned}
$$

The definition (7.1.1) is perhaps suggestive of why, in the language of signal processing, the Weyl and Kohn-Nirenberg transforms are "time-varying filters."

It can be shown that with $k, \sigma, \tau \in L^{2}\left(\mathbb{R}^{2}\right)$, the operators $A_{k}, L_{\sigma}, K_{\sigma}$ are Hilbert-Schmidt, and in fact, the mappings $k \mapsto A_{k}, \sigma \mapsto L_{\sigma}$, and $\tau \mapsto K_{\tau}$ are each isometric isomorphisms of $L^{2}\left(\mathbb{R}^{2}\right)$ onto the space $\mathcal{I}_{2}$ of HilbertSchmidt operators on $L^{2}(\mathbb{R})$. This is well-known for the case of integral operators, and extends easily to the Weyl and Kohn-Nirenberg transforms by an application of the Plancherel formula [23].

Our goal in this chapter is to attempt to illustrate the natural connection between Gabor analysis and integral or pseudodifferential operators, and to demonstrate in particular that Gabor frames provide a convenient and simple tool for analyzing certain properties of these operators. We will not present an exhaustive study of operator theory or its literature. Our exposition is based directly on the joint work of the author with Ramanathan and Topiwala in [15] and with Gröchenig in [14]. Certainly, the connection between pseudodifferential operators and time-frequency analysis is not new, as can be seen, for example, in [11], [19] and many other articles. Our purpose here is simply to provide a clear and self-contained account of a technique from [15], [14] which employs Gabor frames to obtain an interesting result on the spectral properties of pseudodifferential operators. Our proof here is simpler, and extends the result to kernels of integral operators. Other results from [15], [14] that we will not discuss include sufficient conditions for boundedness of pseudodifferential operators acting on 
$L^{2}(\mathbb{R})$ and on the modulation spaces, which are a class of function spaces naturally associated to Gabor analysis.

Let us briefly and incompletely mention some other recent papers which also employ time-frequency expansions in some way. Tachizawa [28], [29] had earlier used Wilson basis expansions to obtain boundedness results for pseudodifferential operators on the modulation spaces. Rochberg and Tachizawa employed Gabor frame expansions in [24], as do Czaja and Rzeszotnik in [2]. Labate applied Gabor analysis to derive results on compositions and compactness of pseudodifferential operators on the modulation spaces in [20], [21]. The above papers are relatively closely related to this chapter; for a survey of much deeper ideas and techniques relating Gabor and wavelet expansions to operator theory we recommend the paper of Meyer [22].

For simplicity of presentation, we will mostly confine our discussion in this chapter to the one-dimensional setting, but extensions to higher dimensions are straightforward. Unlike many approaches to pseudodifferential operators, the result obtained here is easily transferable between the Weyl and Kohn-Nirenberg correspondences.

\subsection{Discussion and Statement of Results}

Given a symbol $\sigma \in L^{2}\left(\mathbb{R}^{2}\right)$, its Weyl transform $L_{\sigma}$ is a Hilbert-Schmidt operator on $L^{2}(\mathbb{R})$. In particular, it is compact, so has a countable set of nonnegative singular values $\left\{s_{j}\left(L_{\sigma}\right)\right\}_{j=1}^{\infty}$, which we arrange in nonincreasing order. These can be defined by spectral theory as the square-roots of the eigenvalues of the positive, self-adjoint operator $L_{\sigma}^{*} L_{\sigma}$, i.e.,

$$
s_{j}\left(L_{\sigma}\right)=\sqrt{\lambda_{j}\left(L_{\sigma}^{*} L_{\sigma}\right)} .
$$

Alternatively, since $L^{2}(\mathbb{R})$ is a Hilbert space, the singular values of $L_{\sigma}$ coincide with its approximation numbers, which are defined in terms of best approximations of $L_{\sigma}$ by finite-rank operators. Specifically,

$$
s_{j}\left(L_{\sigma}\right)=\inf \left\{\left\|L_{\sigma}-T\right\|: \operatorname{rank}(T)<j\right\}, \quad j=1,2,3, \ldots,
$$

where $\|L\|$ denotes the operator norm of an operator $L$.

The Schatten class $\mathcal{I}_{p}$ is the Banach space of all compact operators $K: L^{2}(\mathbb{R}) \rightarrow L^{2}(\mathbb{R})$ whose singular values lie in $\ell^{p}$, with norm

$$
\|K\|_{\mathcal{I}_{p}}=\left(\sum_{j=1}^{\infty} s_{j}(K)^{p}\right)^{1 / p} .
$$

In particular, $\mathcal{I}_{2}$ is the space of Hilbert-Schmidt operators, and $\mathcal{I}_{1}$ is the space of trace-class operators. 
The Weyl correspondence $\sigma \mapsto L_{\sigma}$ defines an isometric isomorphism of $L^{2}\left(\mathbb{R}^{2}\right)$ onto $\mathcal{I}_{2}$, but no similar characterization exists for $\mathcal{I}_{1}$. Daubechies $[3]$ and Hörmander [17] independently derived a sufficient condition on $\sigma$ which implies that its Weyl transform $L_{\sigma}$ is trace-class. Let $L_{s}^{2}\left(\mathbb{R}^{2}\right)$ be the weighted $L^{2}$ space defined by the norm

$$
\|\sigma\|_{L_{s}^{2}}^{2}=\iint|\sigma(x, \xi)|^{2}\left(1+x^{2}+\xi^{2}\right)^{s} d x d \xi,
$$

and let $H^{s}\left(\mathbb{R}^{2}\right)$ be the Sobolev space defined by the norm

$$
\|\sigma\|_{H^{s}}^{2}=\iint|\hat{\sigma}(p, q)|^{2}\left(1+p^{2}+q^{2}\right)^{s} d p d q,
$$

where $\hat{\sigma}$ is the Fourier transform of $\sigma$. Let

$$
\mathcal{H}^{s}\left(\mathbb{R}^{2}\right)=L_{s}^{2}\left(\mathbb{R}^{2}\right) \cap H^{s}\left(\mathbb{R}^{2}\right),
$$

with norm

$$
\|\sigma\|_{\mathcal{H}^{s}}=\|\sigma\|_{L_{s}^{2}}+\|\sigma\|_{H^{s}} .
$$

This is a subspace of $L^{2}\left(\mathbb{R}^{2}\right)$ for $s \geq 0$. We will give another formulation of $\mathcal{H}^{s}\left(\mathbb{R}^{2}\right)$ in terms of modulation spaces in Section 7.3 below.

An equivalent form of the one-dimensional version of the result of Daubechies and Hörmander can now be stated as follows.

Theorem 7.2.1. If $\sigma \in \mathcal{H}^{s}\left(\mathbb{R}^{2}\right)$ with $s>2$, then $L_{\sigma}$ is trace-class.

The higher-dimensional version of this result is that $L_{\sigma}$ is trace-class if $\sigma \in \mathcal{H}^{s}\left(\mathbb{R}^{2 d}\right)$ with $s>2 d$.

We will prove the following result, which extends [15, Prop. 5.4] and [14, Thm. 1.2].

Theorem 7.2.2. Let $s \geq 0$ be given. Let $k, \sigma, \tau \in L^{2}\left(\mathbb{R}^{2}\right)$ define the same operator, i.e., $L=A_{k}=L_{\sigma}=K_{\tau}$. If any one of $k, \sigma, \tau$ lies in $\mathcal{H}^{s}\left(\mathbb{R}^{2}\right)$, then so do the others, and in this case

$$
s_{j}(L)=\mathcal{O}\left(j^{-\frac{s}{2}-\frac{1}{2}}\right) .
$$

Consequently, $L \in \mathcal{I}_{p}$ for $p>\frac{2}{s+1}$, and in particular is trace-class if $s>1$.

The higher-dimensional version of this result is that $s_{j}(L)=\mathcal{O}\left(j^{-\frac{s}{2 d}-\frac{1}{2}}\right)$ if $k, \sigma$, or $\tau$ lies in $\mathcal{H}^{s}\left(\mathbb{R}^{2 d}\right)$, and, in particular, $L$ is trace-class for $s>$ $d$. Given the simplicity of Theorem 7.2.2 and its proof, we would not be surprised if alternative proofs may be found elsewhere in the literature.

\subsection{The Modulation Spaces}

In this section we will give an equivalent formulation of the space $\mathcal{H}^{s}\left(\mathbb{R}^{2}\right)$ in terms of the modulation spaces, which are function spaces naturally associated with Gabor analysis. 
We define translation and modulation of functions of one variable by

$$
T_{a} f(x)=f(x-a) \text { and } M_{b} f(x)=e^{2 \pi i b x} f(x),
$$

and translation and modulation of functions of two variables by

$$
T_{(a, b)} F(x, y)=F(x-a, y-b) \quad \text { and } \quad M_{(c, d)} F(x, y)=e^{2 \pi(c x+d y)} F(x, y)
$$

respectively. The inner product of $f, g \in L^{2}(\mathbb{R})$ is $\langle f, g\rangle=\int f(x) \overline{g(x)} d x$ and similarly the inner product of $F, G \in L^{2}\left(\mathbb{R}^{2}\right)$ is

$$
\langle F, G\rangle=\iint F(x, y) \overline{G(x, y)} d x d y .
$$

Let $\Phi \in L^{2}\left(\mathbb{R}^{2}\right)$ be a fixed nonzero function, which we call a window function. It is well-known that a function $F(x, y)$ lies in $L^{2}\left(\mathbb{R}^{2}\right)$ if and only if its Gabor coefficients $\left\langle F, M_{(c, d)} T_{(a, b)} \Phi\right\rangle$ are square-integrable as a function of $a, b, c, d$. In fact, if we normalize so that $\|\Phi\|_{L^{2}}=1$, then

$$
\iiint \int\left|\left\langle F, M_{(c, d)} T_{(a, b)} \Phi\right\rangle\right|^{2} d a d b d c d d=\|F\|_{L^{2}}^{2},
$$

cf. [16, Prop. 3.2.4]. Thus, membership of a function $F$ in $L^{2}\left(\mathbb{R}^{2}\right)$ is characterized by a norm condition on its Gabor coefficients.

The modulation spaces are likewise defined to be spaces of functions or distributions whose membership in the space is determined by a particular norm condition on the Gabor coefficients. The modulation spaces we will need are the spaces $M_{p, p}^{s}\left(\mathbb{R}^{2}\right)$, which consist of all tempered distributions for which the norm

$$
\|F\|_{M_{p, p}^{s}}^{p}=\iiint \int\left|\left\langle F, M_{(c, d)} T_{(a, b)} \Phi\right\rangle\right|^{p} w_{s}(a, b, c, d)^{p} d a d b d c d d
$$

is finite, where

$$
w_{s}(a, b, c, d)=\left(1+a^{2}+b^{2}+c^{2}+d^{2}\right)^{s / 2} .
$$

In this chapter, we will be most interested in the cases $p=1$ and $p=2$. $M_{p, p}^{s}\left(\mathbb{R}^{2}\right)$ is a subspace of $L^{2}\left(\mathbb{R}^{2}\right)$ for $1 \leq p \leq 2$ and $s \geq 0$.

A large class of window functions $\Phi$, including all the Schwartz-class functions, can be used in (7.3.2) to define the modulation space norm. In particular, each such choice of $\Phi$ yields the same space $M_{p, p}^{s}\left(\mathbb{R}^{2}\right)$, and the different norms gives by (7.3.2) for the different choices of $\Phi$ are all equivalent. More general modulation spaces $M_{p, q}^{s}\left(\mathbb{R}^{d}\right)$ can be defined by placing an $L^{p}$-norm on the translation parameters and an $L^{q}$-norm on the modulation parameters, or other weights $w$ can be used to define analogous modulation spaces $M_{p, q}^{w}\left(\mathbb{R}^{d}\right)$. We refer to [13] for detailed background on the modulation spaces. 
The modulation spaces we will make use of are $M_{1,1}^{0}\left(\mathbb{R}^{2}\right)$ and $M_{2,2}^{s}\left(\mathbb{R}^{2}\right)$ for $s \geq 0$. The space $M_{1,1}^{0}\left(\mathbb{R}^{2}\right)$, sometimes known as the Feichtinger algebra, is a particularly elegant space with many interesting properties, cf. [10], [13]. Clearly, $M_{2,2}^{0}\left(\mathbb{R}^{2}\right)=L^{2}\left(\mathbb{R}^{2}\right)=\mathcal{H}^{0}\left(\mathbb{R}^{2}\right)$. For our purposes, the following identification will be important, cf. [13, Prop. 11.3.1].

Lemma 7.3.1. For $s \geq 0$ we have $M_{2,2}^{s}\left(\mathbb{R}^{2}\right)=\mathcal{H}^{s}\left(\mathbb{R}^{2}\right)$, with equivalent norms.

\subsection{Invariance Properties of the Modulation Space}

In this section we will consider the relationship between the kernel $k$, the Weyl symbol $\sigma$, and the Kohn-Nirenberg symbol $\tau$ of a given operator. We will show that if any one of these lies in the modulation space $M_{2,2}^{s}\left(\mathbb{R}^{2}\right)$, then so do the others. Consequently, when we later prove Theorem 7.2.2, we will be able to restrict our attention to the kernel alone, as the other statements then follow from the invariance of $M_{2,2}^{s}\left(\mathbb{R}^{2}\right)$ under the change from kernel to symbols. For more extensive results on the invariance properties of modulation spaces in connection to pseudodifferential operator theory, we refer to [9].

Considering Definition 7.1.1, we see that if we are given a symbol $\sigma$ which determines the operator $L_{\sigma}$, then the kernel $k$ which yields the same operator is

$$
\begin{aligned}
k(x, y) & =\int \sigma\left(\frac{x+y}{2}, \xi\right) e^{2 \pi i(x-y) \xi} d \xi \\
& =\mathcal{F}_{2}^{-1} \sigma\left(\frac{x+y}{2}, x-y\right) \\
& =\left(\mathcal{F}_{2}^{-1} \sigma \circ M\right)(x, y),
\end{aligned}
$$

where $\mathcal{F}_{2}$ denotes the partial Fourier transform in the second variable defined by

$$
\mathcal{F}_{2} F(x, y)=\int e^{-2 \pi i \xi y} F(x, \xi) d \xi,
$$

and $M$ is the measure-preserving change of variables given by

$$
M(x, y)=\left(\frac{x+y}{2}, x-y\right) .
$$

Thus, the kernel and the Weyl symbol are related by the formulas

$$
k=\mathcal{F}_{2}^{-1} \sigma \circ M \quad \text { and } \quad \sigma=\mathcal{F}_{2}\left(k \circ M^{-1}\right) .
$$

Similarly, the relationship between the kernel $k$ and the Kohn-Nirenberg symbol $\tau$ is

$$
k=\mathcal{F}_{2}^{-1} \tau \circ N \quad \text { and } \quad \tau=\mathcal{F}_{2}\left(k \circ N^{-1}\right)
$$


where $N(x, y)=(x, x-y)$. Therefore, we just have to show that $M_{2,2}^{s}\left(\mathbb{R}^{2}\right)$ is invariant under the transformations above. In fact, this holds more generally for $M_{p, p}^{s}\left(\mathbb{R}^{2}\right)$.

Proposition 7.4.1. $M, N$, and $\mathcal{F}_{2}$ map $M_{p, p}^{s}\left(\mathbb{R}^{2}\right)$ onto itself for $1 \leq p \leq$ $\infty$ and $s \geq 0$. Consequently, if one of $k, \sigma$, or $\tau$ lies in $M_{p, p}^{s}\left(\mathbb{R}^{2}\right)$, then so do the others.

Proof. We will use the norm defined by (7.3.2), keeping in mind that each different choice of window $\Phi$ yields an equivalent norm for $M_{p, p}^{s}\left(\mathbb{R}^{2}\right)$. It is easy to check that $M_{p, p}^{s}\left(\mathbb{R}^{2}\right)$ is invariant under the change of variables given by $M$ or $N$, so it suffices to prove that $M_{p, p}^{s}\left(\mathbb{R}^{2}\right)$ is invariant under the partial Fourier transform $\mathcal{F}_{2}$. If we fix a particular Schwartz-class window function $\Phi$, then it follows from a direct calculation that

$$
\begin{aligned}
\left\langle\mathcal{F}_{2} F, M_{(c, d)} T_{(a, b)} \Phi\right\rangle & =\left\langle F, \mathcal{F}_{2}^{-1} M_{(c, d)} T_{(a, b)} \Phi\right\rangle \\
& =e^{-2 \pi i b d}\left\langle F, M_{(c, b)} T_{(a,-d)}\left(\mathcal{F}_{2}^{-1} \Phi\right)\right\rangle .
\end{aligned}
$$

Therefore, if we let $\Psi=\mathcal{F}_{2}^{-1} \Phi$, then this is just another Schwartz-class window function that can be used to define an equivalent norm for $M_{p, p}^{s}\left(\mathbb{R}^{2}\right)$. Hence,

$$
\begin{aligned}
\left\|\mathcal{F}_{2} F\right\|_{M_{p, p}^{s}}^{p} & =\iiint \int\left|\left\langle\mathcal{F}_{2} F, M_{(c, d)} T_{(a, b)} \Phi\right\rangle\right|^{p} w_{s}(a, b, c, d)^{p} d a d b d c d d \\
& =\iiint \int\left|\left\langle F, M_{(c, b)} T_{(a,-d)} \Psi\right\rangle\right|^{p} w_{s}(a, b, c, d)^{p} d a d b d c d d \\
& \asymp\|F\|_{M_{p, p}^{s}}^{p} .
\end{aligned}
$$

\subsection{Gabor Frames}

We will construct a convenient Gabor frame for $L^{2}\left(\mathbb{R}^{2}\right)$ in this section. We begin first in one dimension, and then move to two dimensions.

For the remainder of this chapter, we let $\phi$ be any particular infinitely differentiable function on $\mathbb{R}$ which is supported in $[0,1]$ and which satisfies

$$
\sum_{n \in \mathbb{Z}}\left|\phi\left(x-\frac{n}{2}\right)\right|^{2}=1 .
$$

The Gabor system generated by $\phi$ with translation parameter $\alpha=1 / 2$ and modulation parameter $\beta=1$ is the collection

$$
\left\{\phi_{m n}\right\}_{m, n \in \mathbb{Z}}=\left\{M_{m} T_{n / 2} \phi\right\}_{m, n \in \mathbb{Z}}=\left\{e^{2 \pi i m x} \phi\left(x-\frac{n}{2}\right)\right\}_{m, n \in \mathbb{Z}} .
$$

It is easy to show that with the hypotheses we have placed on $\phi$, this Gabor system is a normalized tight frame for $L^{2}(\mathbb{R})$, i.e.,

$$
\forall f \in L^{2}(\mathbb{R}), \quad\|f\|_{L^{2}}^{2}=\sum_{m, n}\left|\left\langle f, \phi_{m n}\right\rangle\right|^{2},
$$


cf. [16, Thm. 4.1.2]. This is a kind of discrete, one-dimensional version of (7.3.1). We refer to [5], [13], or [16] for background information on frames and Gabor systems. It follows from (7.5.1) that we have the frame expansions

$$
\forall f \in L^{2}(\mathbb{R}), \quad f=\sum_{m, n}\left\langle f, \phi_{m n}\right\rangle \phi_{m n},
$$

with unconditional convergence of the series in $L^{2}$-norm. The particular choice of $\phi, \alpha, \beta$ made above will be convenient for us, but other Gabor frames would serve as well.

To obtain a frame for $L^{2}\left(\mathbb{R}^{2}\right)$, we can simply form tensor products. That is, we set

$$
\Phi(x, y)=\phi(x) \overline{\phi(y)}
$$

(the purpose of the complex conjugate on $\phi(y)$ in (7.5.2) will become clear in equation (7.6.3) below), and consider the two-dimensional Gabor system

$$
\begin{aligned}
\left\{\Phi_{m n u v}\right\}_{m, n, u, v \in \mathbb{Z}} & =\left\{M_{(m, u)} T_{(n / 2, v / 2)} \Phi\right\}_{m, n, u, v \in \mathbb{Z}} \\
& =\left\{\phi_{m n}(x) \overline{\phi_{u v}(y)}\right\}_{m, n, u, v \in \mathbb{Z}} .
\end{aligned}
$$

This is a normalized tight frame for $L^{2}\left(\mathbb{R}^{2}\right)$, i.e.,

$$
\forall F \in L^{2}\left(\mathbb{R}^{2}\right), \quad\|F\|_{L^{2}}^{2}=\sum_{m, n, u, v}\left|\left\langle F, \Phi_{m n u v}\right\rangle\right|^{2},
$$

and we have corresponding frame expansions [15, Lemma 3.2].

A fundamental fact is that Gabor frames for $L^{2}\left(\mathbb{R}^{2}\right)$ with suitably welllocalized windows (of which $\Phi$ is one) are also frames for the modulation spaces. Specifically, just as (7.5.3) is a discrete version of (7.3.1) characterizing the $L^{2}$-norm in terms of Gabor coefficients, the following is a discrete version of (7.3.2), characterizing the $M_{p, p}^{s}$-norm by Gabor coefficients [13, Cor. 12.2.6].

Lemma 7.5.1. If $1 \leq p<\infty$, then an equivalent norm for $M_{p, p}^{s}\left(\mathbb{R}^{2}\right)$ is given by

$$
\|F\|_{M_{p, p}^{s}}=\sum_{m, n, u, v}\left|\left\langle F, \Phi_{m n u v}\right\rangle\right|^{p} w_{s}(m, n, u, v)^{p} .
$$

\subsection{An Easy Trace-Class Result}

In this section we will show how a Gabor frame expansion can be used to give a simple proof and extension of Theorem 7.2.1. Unfortunately, this approach, which is due to Feichtinger and Gröchenig [12], is not sufficient to prove Theorem 7.2.2. Therefore, in the next section we present a somewhat more sophisticated approach to estimating singular values. With either 
approach, because of the invariance properties of the modulation spaces presented in Proposition 7.4.1, it is sufficient to consider the case of an integral operator $A_{k}$ determined by a kernel $k$. Once the result is established for $A_{k}$, Proposition 7.4.1 implies that it transfers immediately to both the Weyl transform $L_{\sigma}$ and the Kohn-Nirenberg transform $K_{\tau}$.

In Section 7.5 we constructed a normalized tight frame whose elements are $\Phi_{m n u v}(x, y)=\phi_{m n}(x) \overline{\phi_{u v}}(y)$. Hence, if we fix $k \in L^{2}\left(\mathbb{R}^{2}\right)$ then we have the frame expansion

$$
k=\sum_{m, n, u, v}\left\langle k, \Phi_{m n u v}\right\rangle \Phi_{m n u v} .
$$

This gives a corresponding representation of the Hilbert-Schmidt operator $A_{k}$ as

$$
A_{k}=\sum_{m, n, u, v}\left\langle k, \Phi_{m n u v}\right\rangle A_{\Phi_{m n u v}}
$$

with convergence of the series in (7.6.2) in Hilbert-Schmidt norm. Moreover, the operators $A_{\Phi_{m n u v}}$ with kernels $\Phi_{m n u v}$ appearing on the right-hand side of (7.6.2) are rank-one (i.e., have one-dimensional ranges), because

$$
\begin{aligned}
A_{\Phi_{\text {mnuv }}} f(x) & =\int \Phi_{m n u v}(x, y) f(y) d y \\
& =\int \phi_{m n}(x) \overline{\phi_{u v}(y)} f(y) d y \\
& =\left\langle f, \phi_{u v}\right\rangle \phi_{m n}(x) .
\end{aligned}
$$

Thus, (7.6.2) expresses $A_{k}$ as a superposition of rank-one operators. Every rank-one operator is trivially trace-class. Indeed, because the rank is one, $A_{\Phi_{\text {mnuv }}}$ has only a single nonzero singular value. Moreover, since

$$
A_{\Phi_{m n u v}}^{*} A_{\Phi_{m n u v}} f=\left\|\phi_{m n}\right\|_{L^{2}}^{2}\left\langle f, \phi_{u v}\right\rangle \phi_{u v}
$$

the trace-class norm of $A_{\Phi_{m n u v}}$ is

$$
\begin{aligned}
\left\|A_{\Phi_{m n u v}}\right\|_{\mathcal{I}_{1}} & =s_{1}\left(A_{\Phi_{m n u v}}\right) \\
& =\sqrt{\lambda_{1}\left(A_{\Phi_{m n u v}}^{*} A_{\Phi_{m n u v}}\right)} \\
& =\left\|\phi_{u v}\right\|_{L^{2}}\left\|\phi_{m n}\right\|_{L^{2}} \\
& =\|\phi\|_{L^{2}}^{2},
\end{aligned}
$$

which is a constant independent of $m, n, u, v$. Taking the trace-class norm of both sides of (7.6.2), we therefore have

$$
\left\|A_{k}\right\|_{\mathcal{I}_{1}} \leq \sum_{m, n, u, v}\left|\left\langle k, \Phi_{m n u v}\right\rangle\right|\left\|A_{\Phi_{m n u v}}\right\|_{\mathcal{I}_{1}}=\|\phi\|_{L^{2}}^{2} \sum_{m, n, u, v}\left|\left\langle k, \Phi_{m n u v}\right\rangle\right| .
$$


Recalling from Lemma 7.5.1 the norm for the modulation space $M_{1,1}^{0}\left(\mathbb{R}^{2}\right)$, we conclude that if

$$
\|k\|_{M_{1,1}^{0}}=\sum_{m, n, u, v}\left|\left\langle k, \Phi_{m n u v}\right\rangle\right|<\infty
$$

then (7.6.2) converges absolutely in trace-class norm, and hence $A_{k}$ itself is trace-class. Combining with the invariance properties of $M_{1,1}^{0}\left(\mathbb{R}^{2}\right)$ given in Proposition 7.4.1, this gives the following result extending [12, Thm. 3].

Proposition 7.6.1. Let $k, \sigma, \tau \in L^{2}(\mathbb{R})$ define the same operator, i.e., $L=A_{k}=L_{\sigma}=K_{\tau}$. If any one of $k, \sigma, \tau$ lies in $M_{1,1}^{0}\left(\mathbb{R}^{2}\right)$, then $L$ is trace-class.

To compare Proposition 7.6.1 to Theorem 7.2.2, we note the following special case of an embedding result of Gröchenig [12].

Proposition 7.6.2. $M_{2,2}^{s}\left(\mathbb{R}^{2}\right) \subset M_{1,1}^{0}\left(\mathbb{R}^{2}\right)$ exactly for $s>2$.

Hence, the combination of Propositions 7.6.1 and 7.6.2 already give an improvement to Theorem 7.2.1, but do not recover Theorem 7.2.2. In particular, the hypothesis of Proposition 7.6.1 that $k \in M_{1,1}^{0}\left(\mathbb{R}^{2}\right)$ implies that (7.6.2) converges absolutely in trace-class norm. This is a very strong requirement, and therefore it is not surprising that Proposition 7.6.1 is not the best possible. In the next section, we present a different approach to estimating singular values which does not require the assumption of absolute convergence of (7.6.2).

Note added. Following completion of this chapter, J. Toft brought to our attention the paper [26] by J. Sjöstrand. Sjöstrand introduces in that paper a space which in our terminology is the modulation space $M_{\infty, 1}^{0}\left(\mathbb{R}^{2 d}\right)$ and proves, among other results, that if a symbol lies in $M_{\infty, 1}^{0}\left(\mathbb{R}^{2 d}\right)$ then the corresponding pseudodifferential operator is bounded on $L^{2}\left(\mathbb{R}^{d}\right)$. This is a special case of the independent but later results in [15], [14], [13]. Additionally, Sjöstrand mentions in [26], without proof, a trace-class result, and proves in [6, Sec. 9] that (in our terminology) if $\sigma$ or $\tau$ lies in $M_{1,1}^{0}\left(\mathbb{R}^{2 d}\right)$, then the corresponding operators $L_{\sigma}$ or $K_{\tau}$ are trace-class, compare Proposition 7.6.1.

\subsection{Finite-Rank Approximations}

To prove Theorem 7.2.2, we need to estimate the singular values of $A_{k}$, and to do this we construct finite-rank approximations based on a frame expansion of the kernel $k$. Because of the invariance properties presented in Proposition 7.4.1, it suffices to consider the kernel alone, as the result then transfers immediately to both the Weyl symbol $\sigma$ and the Kohn-Nirenberg symbol $\tau$. 
As in the preceding section, we begin with a kernel $k \in L^{2}\left(\mathbb{R}^{2}\right)$ and consider its expansion with respect to the normalized tight frame whose elements are $\Phi_{m n u v}(x, y)=\phi_{m n}(x) \overline{\phi_{u v}(y)}$. We have

$$
k=\sum_{m, n, u, v}\left\langle k, \Phi_{m n u v}\right\rangle \Phi_{m n u v} .
$$

Then, for each integer $N>0$, we define an approximation $k_{N}$ to $k$ by truncating the sum in (7.7.1). Specifically, we define

$$
k_{N}=\sum_{(m, n, u, v) \in B_{N}}\left\langle k, \Phi_{m n u v}\right\rangle \Phi_{m n u v}
$$

where

$$
B_{N}=\left\{(m, n, u, v) \in \mathbb{Z}^{4}:|m|,|n|,|u|,|v| \leq N\right\}=\{-N, \ldots, N\}^{4} .
$$

The integral operator with kernel $k_{N}$ is

$$
A_{k_{N}}=\sum_{(m, n, u, v) \in B_{N}}\left\langle k, \Phi_{m n u v}\right\rangle A_{\Phi_{m n u v}} .
$$

We need to estimate the rank of $A_{k_{N}}$. Since $A_{\Phi_{\text {mnuv }}}$ is rank-one and since $B_{N}$ contains $(2 N+1)^{4}$ points, we certainly have from (7.7.2) that $\operatorname{rank}\left(A_{k_{N}}\right) \leq(2 N+1)^{4}$. However, this naive estimate is much too large. From (7.6.3), we see that the range of $A_{\Phi_{m n u v}}$ is the line through $\phi_{m n}$. Therefore, the rank of $A_{k_{N}}$ is bounded by the number of distinct values of $(m, n)$ in the sum in $(7.7 .2)$, which is $(2 N+1)^{2}$. Thus,

$$
\operatorname{rank}\left(A_{k_{N}}\right) \leq(2 N+1)^{2}<10 N^{2} .
$$

Consequently, from the definition of singular values given in (7.2.1), we have that

$$
s_{10 N^{2}}\left(A_{k}\right) \leq\left\|A_{k}-A_{k_{N}}\right\| .
$$

By re-indexing and using the fact that the singular values are nonincreasing, we will be able to convert this into an estimate for all the singular values if we are able to estimate the operator norm $\left\|A_{k}-A_{k_{N}}\right\|$.

Now, we know that the Hilbert-Schmidt norm of $A_{k}-A_{k_{N}}$ equals the $L^{2}$-norm of $k-k_{N}$. Since the operator norm is bounded by the HilbertSchmidt norm, we therefore have

$$
\left\|A_{k}-A_{k_{N}}\right\| \leq\left\|A_{k}-A_{k_{N}}\right\|_{\mathcal{I}_{2}}=\left\|k-k_{N}\right\|_{L^{2}} .
$$

To estimate this quantity, we use the fact that $\left\{\Phi_{m n u v}\right\}_{m, n, u, v \in \mathbb{Z}}$ is a normalized tight frame. In particular, we have the inequality

$$
\left\|\sum_{m, n, u, v} c_{m n u v} \Phi_{m n u v}\right\|_{L^{2}}^{2} \leq \sum_{m, n, u, v}\left|c_{m n u v}\right|^{2}
$$


for any coefficients $c_{m n u v}$. Therefore,

$$
\begin{aligned}
\left\|k-k_{N}\right\|_{L^{2}}^{2} & =\left\|\sum_{(m, n, u, v) \notin B_{N}}\left\langle k, \Phi_{m n u v}\right\rangle \Phi_{m n u v}\right\|_{L^{2}}^{2} \\
& \leq \sum_{(m, n, u, v) \notin B_{N}}\left|\left\langle k, \Phi_{m n u v}\right\rangle\right|^{2} .
\end{aligned}
$$

Our problem has thus reduced to one of estimating the size of the Gabor frame coefficients $\left\langle k, \Phi_{\text {mnuv }}\right\rangle$ outside of the box $B_{N}$. This is in fact exactly the problem addressed in the classic energy concentration theorem of Daubechies [4, Thm. 3.1], and we could use that result to estimate (7.7.3). However, we can proceed even more directly by using the fact that (7.5.4) is an equivalent norm for $\mathcal{H}^{s}\left(\mathbb{R}^{2}\right)=M_{2,2}^{s}\left(\mathbb{R}^{2}\right)$. In particular, if $k \in M_{2,2}^{s}\left(\mathbb{R}^{2}\right)$, then we have

$$
\begin{aligned}
\left(s_{10 N^{2}}\left(A_{k}\right)\right)^{2} & \leq\left\|A_{k}-A_{k_{N}}\right\|_{\mathcal{I}_{2}}^{2} \\
& =\left\|k-k_{N}\right\|_{L^{2}}^{2} \\
& \leq \sum_{(m, n, u, v) \notin B_{N}}\left|\left\langle k, \Phi_{m n u v}\right\rangle\right|^{2} \frac{w_{s}(m, n, u, v)^{2}}{w_{s}(m, n, u, v)^{2}} \\
& \leq \frac{1}{\left(1+N^{2}\right)^{s}} \sum_{(m, n, u, v) \notin B_{N}}\left|\left\langle k, \Phi_{m n u v}\right\rangle\right|^{2} w_{s}(m, n, u, v)^{2} \\
& \leq N^{-2 s}\|k\|_{M_{2,2}^{s}}^{2} .
\end{aligned}
$$

Hence, for any positive integer $j$ of the form $j=10 N^{2}$, we have shown that

$$
s_{j}\left(A_{k}\right) \leq C_{1} j^{-s / 2} .
$$

Because the singular values are nonincreasing, this estimate can be extended to all of the singular values (at the cost of replacing $C_{1}$ in (7.7.5) by a larger constant). In particular, given an arbitrary positive integer $j$, let $N$ be such that $10 N^{2} \leq j<10(N+1)^{2}$. Then

$$
\begin{aligned}
s_{j}\left(A_{k}\right) \leq s_{10 N^{2}}\left(A_{k}\right) & \leq C_{1}\left(10 N^{2}\right)^{-s / 2} \\
& =C_{1} 2^{s}\left(10(2 N)^{2}\right)^{-s / 2} \\
& \leq C_{1} 2^{s}\left(10(N+1)^{2}\right)^{-s / 2} \\
& \leq C_{1} 2^{s} j^{-s / 2} .
\end{aligned}
$$

Thus we have shown that

$$
k \in \mathcal{H}^{s}\left(\mathbb{R}^{2}\right) \Longrightarrow s_{j}\left(A_{k}\right) \in \mathcal{O}\left(j^{-s / 2}\right) .
$$

However, this is not yet the decay rate claimed in Theorem 7.2.2! 


\subsection{Improving the Estimate}

We will use an idea due to Weyl to improve the estimate in (7.7.7). The following result was proved in [31, Satz I] for the case of eigenvalues of a self-adjoint operators, and was later extended to the case of singular values. The following is sometimes known as Fan's inequality [8], cf. [1, p. 213], [7, p. 1089], [25, Thm. 1.7]. Many other interesting inequalities and results can be found in [1] and [25].

Lemma 7.8.1. If $L_{1}, L_{2}$ are compact operators on $L^{2}(\mathbb{R})$, then

$$
s_{m+n-1}\left(L_{1}+L_{2}\right) \leq s_{m}\left(L_{1}\right)+s_{n}\left(L_{2}\right)
$$

for all integers $m, n>0$.

Using this fact, we obtain the following improvement to (7.2.1) for estimating singular values, cf. [31, Satz III] for the eigenvalue case. Moreover, this result estimates the singular values in terms of the Hilbert-Schmidt norm, which is better suited for our purposes than the operator norm because of the fact that $k \mapsto A_{k}$ is an isometric isomorphism of $L^{2}\left(\mathbb{R}^{2}\right)$ onto the space $\mathcal{I}_{2}$ of Hilbert-Schmidt operators.

Corollary 7.8.1. If $L$ is a Hilbert-Schmidt operator on $L^{2}(\mathbb{R})$, then

$$
\sum_{j \geq n} s_{j}(L)^{2} \leq \inf \left\{\|L-T\|_{\mathcal{I}_{2}}^{2}: \operatorname{rank}(T)<n\right\} .
$$

Proof. Suppose that $\operatorname{rank}(T)<n$ and that $j \geq n$. Then

$s_{j}(L)=s_{(j-n+1)+n-1}(L-T+T) \leq s_{j-n+1}(L-T)+s_{n}(T)=s_{j-n+1}(L-T)$,

the last equality following from the fact that $s_{n}(T)=0$ since $\operatorname{rank}(T)<n$. Therefore,

$$
\sum_{j \geq n} s_{j}(L)^{2} \leq \sum_{j \geq n} s_{j-n+1}(L-T)^{2}=\sum_{n=1}^{\infty} s_{n}(L-T)^{2}=\|L-T\|_{\mathcal{I}_{2}}^{2} .
$$

Taking the infimum over all over $T$ with $\operatorname{rank}(T)<n$ therefore yields the desired inequality.

As a corollary, we obtain the following improved estimate for the individual singular values.

Corollary 7.8.2. If $L$ is a Hilbert-Schmidt operator on $L^{2}(\mathbb{R})$, then

$$
s_{2 n}(L)^{2} \leq \frac{1}{n} \inf \left\{\|L-T\|_{\mathcal{I}_{2}}^{2}: \operatorname{rank}(T)<n\right\} .
$$


Proof. Since the singular values of $L$ are nonincreasing, we have

$$
\begin{aligned}
n s_{2 n}(L)^{2} \leq \sum_{j=n+1}^{2 n} s_{j}(L)^{2} & \leq \sum_{j \geq n} s_{j}(L)^{2} \\
& \leq \inf \left\{\|L-T\|_{\mathcal{I}_{2}}^{2}: \operatorname{rank}(T)<n\right\}
\end{aligned}
$$

the last inequality following from Corollary 7.8.1.

We can apply this now to the case of an integral operator $A_{k}$ with kernel $k \in \mathcal{H}^{s}\left(\mathbb{R}^{2}\right)$. We use the same finite-rank approximations as in Section 7.7. Since $\operatorname{rank}\left(A_{k_{N}}\right)<10 N^{2}$, we have from (7.8.1) and (7.7.4) that

$$
\begin{aligned}
\left(s_{20 N^{2}}\left(A_{k}\right)\right)^{2} & \leq \frac{1}{10 N^{2}}\left\|A_{k}-A_{k_{N}}\right\|_{\mathcal{I}_{2}}^{2} \\
& \leq \frac{N^{-2 s}\|k\|_{\mathcal{H}^{s}}^{2}}{10 N^{2}} \\
& =C_{2} N^{-2 s-2}\|k\|_{\mathcal{H}^{s}}^{2} .
\end{aligned}
$$

Then, by reindexing as in (7.7.6), we conclude that there is a constant $C_{3}>0$ such that

$$
s_{j}\left(A_{k}\right) \leq C_{3} j^{-\frac{s}{2}-\frac{1}{2}}
$$

for every $j>0$. This proves Theorem 7.2.2.

\subsection{Conclusion and Observations}

It is interesting to note that the fact that $\left\{\Phi_{m n u v}\right\}_{m, n, u, v \in \mathbb{Z}}$ is a $G a$ bor frame enters the argument only a few specific points. Indeed, if we start instead with any collection of functions $\left\{\psi_{m n}\right\}_{m, n \in \mathbb{Z}}$ which forms a normalized tight frame for $L^{2}(\mathbb{R})$ and which satisfies $0<\inf \left\|\psi_{m n}\right\| \leq$ $\sup \left\|\psi_{m n}\right\|<\infty$ and then define

$$
\Psi_{m n u v}(x, y)=\psi_{m n}(x) \overline{\psi_{u v}(y)}
$$

then the same arguments as in Section 7.6 give the following variation on Proposition 7.6.1.

Proposition 7.9.1. If $k \in L^{2}\left(\mathbb{R}^{2}\right)$ is such that

$$
\sum_{m, n, u, v}\left|\left\langle k, \Psi_{m n u v}\right\rangle\right|<\infty
$$

then $A_{k}$ is trace-class.

However, we cannot transfer this result to the Weyl or Kohn-Nirenberg symbols without further information about the frame $\left\{\Psi_{m n u v}\right\}_{m, n, u, v \in \mathbb{Z}}$. 
In particular, the assumption that $\left\{\Phi_{m n u v}\right\}_{m, n, u, v \in \mathbb{Z}}$ was a Gabor frame allowed us to give in Section 7.6 a practical interpretation of the hypothesis $\sum\left|\left\langle k, \Phi_{\text {mnuv }}\right\rangle\right|<\infty$. Specifically, we know that $\sum\left|\left\langle k, \Phi_{\text {mnuv }}\right\rangle\right|$ defines a norm for the concrete function space $M_{1,1}^{0}\left(\mathbb{R}^{2}\right)$, and we know many explicit properties of this space. In particular, we have the invariances given in Proposition 7.4.1 which allow us to interchange between kernel and symbol, and we have the equivalences of Lemma 7.3.1 and the embeddings of Proposition 7.6.2 which allow us to compare Proposition 7.6.1 to Theorem 7.2.1. Similar remarks apply to the analysis in Section 7.7 leading to a proof of Theorem 7.2.2. It would be interesting, then, to see if other practical interpretations of Proposition 7.9.1 could be made by using wavelet or other frames $\left\{\Psi_{m n u v}\right\}_{m, n, u, v \in \mathbb{Z}}$.

\section{Acknowledgments}

The author was supported by NSF Grant DMS-9970524, and gratefully acknowledges helpful comments and discussions regarding this chapter with John Benedetto, Hans Feichtinger, Karlheinz Gröchenig, A.J.E.M. Janssen, Joachim Toft, and David Walnut, and additional conversations related to the subject of pseudodifferential operators with Ryuichi Ashino, Demetrio Labate, Michihiro Nagase, Jay Ramanathan, Pankaj Topiwala, Rémi Vaillancourt, and Man-Wah Wong.

\subsection{REFERENCES}

[1] M. S. Birman and M. Z. Solomjak. "Spectral Theory of SelfAdjoint Operators in Hilbert Space." Translated from the 1980 Russian original by S. Khrushchëv and V. Peller. Mathematics and its Applications (Soviet Series), D. Reidel, Dordrecht (1987).

[2] W. Czaja and Z. Rzeszotnik. Pseudodifferential operators and Gabor frames: Spectral asymptotics. Math. Nachr., 233-234 (2002), pp. 77-88.

[3] I. Daubechies. On the distributions corresponding to bounded operators in the Weyl quantization. Comm. Math. Phys., 75 (1980), pp. 229-238.

[4] I. Daubechies. The wavelet transform, time-frequency localization and signal analysis. IEEE Trans. Inform. Theory, 39 (1990) pp. 961-1005.

[5] I. Daubechies. "Ten Lectures on Wavelets." SIAM, Philadelphia (1992). 
[6] M. Dimassi and J. Sjöstrand, "Spectral Asymptotics in the SemiClassical Limit." London Math. Soc. Lecture Note Series 268, Cambridge University Press, Cambridge (1999).

[7] N. Dunford and J. T. Schwartz. "Linear Operators, Part II." Wiley, New York (1988).

[8] K. Fan. Maximum properties and inequalities for the eigenvalues of completely continuous cperators. Proc. Nat. Acad. Sci., 37 (1951), 760-766.

[9] H. G. Feichtinger and W. Kozek. Quantization of TF latticeinvariant operators on elementary LCA groups. In "Gabor Analysis and Algorithms: Theory and Applications," H. G. Feichtinger and T. Strohmer, eds., Birkhäuser, Boston (1997) pp. 233-266.

[10] H. G. Feichtinger and G. Zimmermann. A Banach space of test functions for Gabor analysis. In "Gabor Analysis and Algorithms: Theory and Applications," H. G. Feichtinger and T. Strohmer, eds., Birkhäuser, Boston (1997) pp. 123-170.

[11] G. B. Folland. "Harmonic Analysis on Phase Space." Ann. of Math. Studies, Princeton University Press, Princeton, NJ (1989).

[12] K. Gröchenig. An uncertainty principle related to the Poisson summation formula. Studia Math., 121 (1996), pp. 87-104.

[13] K. Gröchenig. "Foundations of Time-Frequency Analysis." Birkhäuser, Boston (2001).

[14] K. Gröchenig and C. Heil. Modulation spaces and pseudodifferential operators. Integral Equations Operator Theory, 34 (1999), pp. 439-457.

[15] C. Heil, J. Ramanathan, and P. Topiwala. Singular values of compact pseudodifferential operators. J. Funct. Anal., 150 (1997), pp. $426-452$.

[16] C. E. Heil and D. F. Walnut. Continuous and discrete wavelet transforms. SIAM Review, 31 (1989), pp. 628-666.

[17] L. Hörmander. The Weyl calculus of pseudodifferential operators. Comm. Pure Appl. Math., 32 (1979), pp. 360-444.

[18] L. Hörmander. "The Analysis of Linear Partial Differential Operators, I," Second Edition. Springer-Verlag, Berlin (1990).

[19] R. Howe. Quantum mechanics and partial differential equations. J. Funct. Anal., 38 (1980), pp. 188-254. 
[20] D. Labate. Time-frequency analysis of pseudodifferential operators. Monatshefte Math., 133 (2001), pp. 143-156.

[21] D. Labate. Pseudodifferential operators on modulation spaces. J. Math. Anal. Appl., 262 (2001), pp. 242-255.

[22] Y. Meyer. Wavelets and operators. In "Different Perspectives on Wavelets" (San Antonio, TX, 1993), I. Daubechies, ed., Proc. Sympos. Appl. Math. Vol. 47, Amer. Math. Soc., Providence, RI (1993), pp. 35-58.

[23] J. C. T. Pool. Mathematical aspects of the Weyl correspondence. J. Math. Phys., 7 (1966), pp. 66-76.

[24] R. Rochberg and K. Tachizawa. Pseudodifferential operators, Gabor frames, and local trigonometric bases. In "Gabor Analysis and Algorithms: Theory and Applications," H. G. Feichtinger and T. Strohmer, eds., Birkhäuser, Boston (1997) pp. 171-192.

[25] B. Simon. "Trace ideals and their applications." Cambridge University Press, Cambridge (1979).

[26] J. Sjöstrand, An algebra of pseudodifferential operators. Math. Res. Lett., 1 (1994), pp. 185-192.

[27] E. M. Stein. "Harmonic Analysis: Real-Variable Methods, Orthogonality, and Oscillatory Integrals." Princeton University Press, Princeton, NJ (1993).

[28] K. Tachizawa. The boundedness of pseudodifferential operators on modulation spaces. Math. Nachr., 168 (1994), pp. 263-277.

[29] K. Tachizawa. The pseudodifferential operators and Wilson bases. J. Math. Pures Appl., 75 (1996), pp. 509-529.

[30] M. E. Taylor. "Pseudodifferential Operators." Princeton University Press, Princeton, NJ (1981).

[31] H. Weyl. Das asymptotische Verteilungsgesetz der Eigenwerte linearer partieller Differentialgleichungen (mit einer Anwendung auf die Theorie der Hohlraumstrahlung). Math. Ann., 71 (1911), pp. $441-479$.

[32] M. W. Wong. "Weyl Transforms." Springer-Verlag, New York (1998). 


\section{ERRATA}

Note: This errata is not included in the published version of this paper.

(1) On p. 160 , in the definition of $\Phi_{m n u v}$, replace $M_{(m, u)}$ by $M_{(m,-u)}$, i.e.,

$$
\begin{aligned}
\left\{\Phi_{m n u v}\right\}_{m, n, u, v \in \mathbb{Z}} & =\left\{M_{(m,-u)} T_{(n / 2, v / 2)} \Phi\right\}_{m, n, u, v \in \mathbb{Z}} \\
& =\left\{\phi_{m n}(x) \overline{\phi_{u v}(y)}\right\}_{m, n, u, v \in \mathbb{Z}} .
\end{aligned}
$$

(2) In Lemma 7.5.1, an exponent is missing on the left side of equation (7.5.4). This equation should read:

$$
\|F\|_{M_{p, p}^{s}}^{p}=\sum_{m, n, u, v}\left|\left\langle F, \Phi_{m n u v}\right\rangle\right|^{p} w_{s}(m, n, u, v)^{p} .
$$

